\title{
SYMBOLIC CALCULUS FOR ALGEBRAS OF FOURIER-STIELTJES TRANSFORMS
}

\author{
COLIN C. GRAHAM
}

The purpose of this note is to prove Theorem 1 below. $G$ is a nondiscrete locally compact abelian group with dual group $\Gamma$. The algebra of Fourier transforms on $\Gamma$ is $A(\Gamma)$, and the algebra of FourierStieltjes transforms is $B(\Gamma)$. If $\mu$ is a measure in $G, \hat{\mu}$ is its FourierStieltjes transform, and $\|\hat{\mu}\|$ is the total variation norm $\|\mu\|$.

Theorem 1. Let $X$ be a closed subalgebra of $B(\Gamma)$ such that

(i) $A(\Gamma) \subseteq X$,

(ii) $\hat{\delta}_{0} \in X$ ( $\hat{\delta}_{0}$ is the identity of $B(\Gamma)$ ),

(iii) $X$ contains a real function which is the transform of a measure whose powers are pairwise mutually singular.

Suppose $F$ is a complex valued function defined on $[-1,1]$ and suppose $F \circ f \in B(\Gamma)$ for all $f \in X$ such that $f(\Gamma) \subseteq[-1,1]$. Then $F$ is the restriction to $[-1,1]$ of an entire function.

Once Lemma 6.3 .6 of [2] is extended to our version (Lemma 2 below), the proof of Theorem 1 follows the pattern of $[2,6.3]$. We shall let $F \circ \mu$ be the unique measure having $F \circ \hat{\mu}$ as its transform (when $F \circ \mu$ is defined).

Lemma $1[2,2.6 .7]$. Let $C$ be a compact subset of $\Gamma, \Gamma$ a locally compact group and $\epsilon>0$. Then there is a Fourier transform $f \in A(\Gamma)$ such that

(i) $\|f\| \leqq 1+\epsilon$,

(ii) support $f$ is compact,

(iii) $f$ is one on $C$, and $0 \leqq f \leqq 1$.

Lemma 2. Suppose $F$ is defined on all of the line $R$, and $X$ satisfies the hypotheses of Theorem 1 , and $F \circ f \in B(\Gamma)$ whenever $f$ is a real function in $X$. Then for each real function $\hat{\mu} \in X$ there exist numbers $\eta>0, K$ such that $\nu \in X$ a real function and $\|\nu\|<\eta$ imply

$$
\|F \circ(\mu+\nu)\|<K \text {. }
$$

Proof. If the conclusion is false, then there exist $\mu$ and $\nu_{j} \in X$ such that $\left\|\nu_{j}\right\|<2^{-i}, \hat{\mu}(\Gamma) \subset R, \quad \hat{\nu}_{j}(\Gamma) \subseteq R$, and $\left\|F \circ\left(\mu+\nu_{j}\right)\right\|>j$, $j=1,2,3, \cdots$. We may, in fact, by applying Lemma 1 and $[2,1.9 .1]$ assume that $\nu_{j} \in L^{1}(G)$ and $\hat{\nu}_{j}$ has compact support. Indeed,

Received by the editors February 20, 1969. 
$\left\|F \circ\left(\mu+\nu_{j}\right)\right\|>j$ implies by $[2,1.9 .1]$ that there exists a trigonometric polynomial

$$
P(x)=\sum_{k=1}^{N} c_{k}\left(x, \gamma_{k}\right), \quad \gamma_{k} \in \Gamma
$$

with

$$
\|P\|_{\infty}=\sup \{|P(x)|: x \in G\} \leqq 1
$$

and

$$
\left|\sum_{k} c_{k} F \circ\left(\left(\hat{\mu}+\hat{\nu}_{j}\right)\left(\gamma_{k}\right)\right)\right|>j .
$$

Let $q \in L^{1}(G)$ be chosen by Lemma 1 so that $\|q\|_{1}<2$ and $\hat{q}=1$ on support $\hat{P}\left(=\left\{\gamma_{k}\right\}_{k=1}^{N}\right)$. Then $\left\|F \circ\left(\mu+q * \nu_{j}\right)\right\|>j$.

The basic trick now is to "adjust" the $\nu_{j}$ so that the supports of their transforms are "very" disjoint. That way we can add up the $\nu_{j}$ (since $\left\|\nu_{j}\right\|<2^{-j}$ we get an element of $\left.L^{1}(G)\right), f=\sum \nu_{j}$, and then pick out $F \circ\left(\mu+\nu_{j}\right)$ from $F \circ(\mu+f)$ by multiplying the latter (roughly speaking) by a function $h_{j} \in L^{1}(G)$ such that $\hat{h}_{j}=1$ on the support of $\hat{\nu}_{j}$, but $\hat{h}_{j} \cdot \hat{h}_{k}=0$ if $j \neq k$. We then will get an equality of the form

$$
h_{j} * F \circ(\mu+f)+\left(\delta_{0}-h_{j}\right) * F \circ(\mu)=F \circ\left(\mu+\nu_{j}\right) .
$$

Now take norms and apply the triangle inequality and the multiplication inequality. We obtain

$$
\left\|h_{j}\right\|\|F \circ(\mu+f)\|+\left\|\left(\delta_{0}-h_{j}\right)\right\|\|F \circ(\mu)\| \geqq\left\|F \circ\left(\mu+\nu_{j}\right)\right\|>j .
$$

The $h_{j}$ will be chosen with $\left\|h_{j}\right\|<6$, so we have the absurdity:

$$
6\|F \circ(\mu+f)\|+7\|F \circ(\mu)\|>j \quad \text { for each } j .
$$

Before we give the details of the adjustments we must make to the $\nu_{j}$, we first observe that if the supports of the $\nu_{j}$ all lie in the same compact subset of $\Gamma$, then $\left\|F \circ\left(\mu+\nu_{j}\right)\right\|$ must be bounded. Indeed, let support $\hat{\nu}_{j} \subset C$ for all $j$. Let $g, h \in L^{1}(G)$ be such that support $\hat{g}$ and support $\hat{h}$ are compact, $\|g\|<2,\|h\|<2, \hat{g}=1$ on $C$ and $\hat{h}=1$ on support $\hat{g}$. Then $F \circ\left(\mu+\nu_{j}\right)=g * F \circ\left(\mu+\nu_{j}\right)+\left(\delta_{0}-g\right) * F \circ\left(\mu+\nu_{j}\right)$ $=g * F \circ\left(h * \mu+\nu_{j}\right)+\left(\delta_{0}-g\right) * F \circ \mu$. We now need only show that the term $F \circ\left(h * \mu+\nu_{j}\right)$ is norm-bounded.

Case I. $\Gamma$ discrete. We may use the arguments of $[2,6.3 .4]$ to show that $F$ is continuous. Let $N$ be the number of elements of $C^{\prime}=$ support $\hat{h}$. Then $N$ is finite. Let $L=\sup \left\{|F(z)|: z \in\left(\hat{\mu}+\hat{\nu}_{j}\right)\left(C^{\prime}\right), j=1,2, \cdots\right\}$. 
$L$ is also finite since the sup is over a relatively compact set inside domain $F$. Then

$$
\left\|F \circ\left(h * \mu+\nu_{j}\right)\right\| \leqq N L .
$$

This follows because $h * \mu+\nu_{j}$, and $F \circ\left(h * \mu+\nu_{j}\right)$ are trigonometric polynomials and $G$ is compact.

Case II. $\Gamma$ nondiscrete. Then by $[2,6.5 .1$ and 6.6.1], we may assume $F$ is analytic in a neighborhood of $R$. Now, $h * \mu+\nu_{j} \in L^{1}(G)$. The (Cauchy) formula of Gelfand [1, Theorem 19] applies. Thus $F \circ\left(h * \mu+\nu_{j}\right)$ is norm-bounded.

We are now ready to adjust the $\nu_{j}$. We will obtain functions $g_{j}$ and $h_{j} \in L^{1}(G)$ such that (i) $\left\|g_{j}\right\|_{1}<2^{-j}$, (ii) support $\hat{g}_{j} \subseteq\left\{\hat{h}_{j}=1\right\}$, (iii) $0 \leqq \hat{h}_{j} \leqq 1$, (iv) $\left\|h_{j}\right\|_{1}<6$, (v) support $\hat{h}_{j}$ is compact, (vi) $\left\|F \circ\left(\mu+g_{j}\right)\right\|>j$ and (vii) $h_{j} * h_{k}=0$ if $j \neq k$. We will construct these functions by induction. Let $g_{1}=\nu_{1}$, and pick any $h_{1} \in L^{1}(G)$ satisfying (ii)-(v). One exists by $[2,2.6 .7]$. Now assume we have found $g_{1}, \cdots, g_{n}$ and $h_{1}, \cdots, h_{n}$ satisfying (i)-(vii) for $j, k \leqq n$. We construct $g_{n+1}$ and $h_{n+1}$.

Pick $a, b, c \in L^{1}(G)$ such that $0 \leqq \hat{a}, \hat{b}, \hat{c}, \leqq 1,\|a\|_{1},\|b\|_{1},\|c\|_{1}<2$, and support $\hat{h}_{j} \subseteq\{\hat{a}=1\} \subseteq$ support $a \subseteq\{\hat{b}=1\} \subseteq$ support $b \subseteq\{\hat{c}=1\}$, and support $\hat{c}$ is compact. This can be done by repeated application of Lemma 1.

We claim $\left\|F \circ\left(\mu+\left(\delta_{0}-a\right) * \nu_{j}\right)\right\| \rightarrow \infty$ as $j \rightarrow \infty$. Indeed,

$$
\begin{aligned}
F \circ\left(\mu+\nu_{j}\right)= & b * F \circ\left(\mu+\nu_{j}\right)+\left(\delta_{0}-b\right) * F \circ\left(\mu+\nu_{j}\right) \\
= & b * F \circ\left(c * \mu+c * \nu_{j}\right) \\
& +\left(\delta_{0}-b\right) * F \circ\left(\mu+\left(\delta_{0}-a\right) * \nu_{j}\right) .
\end{aligned}
$$

The first term on the right-hand side of (9) is norm-bounded since support $\hat{c} \hat{v}_{j} \subseteq$ support $\hat{c}$ (which was chosen compact) for all $j$. Therefore, $\left\|F \circ\left(\mu+\left(\delta_{0}-a\right) * \nu_{j}\right)\right\|$ is increasing. Pick $j$ so large that $\left\|F \circ\left(\mu+\left(\delta_{0}-a\right) * \nu_{j}\right)\right\|>n+1$ and such that $\left\|\nu_{j}\right\|<2^{-(n+3)}$. Let $g_{n+1}=\left(\delta_{0}-a\right) * \nu_{j}$. Then support $\hat{g}_{n+1}$ is compact, so there exists $f \in L^{1}(G)$ such that $0 \leqq \hat{f} \leqq 1,\|f\|_{1}<2$, support $\hat{f}$ compact and $\hat{f}=1$ on support $\hat{g}_{n+1}$. Let $h_{n+1}=\left(\delta_{0}-a\right) * f$. Then $g_{1}, \cdots, g_{n+1}$ and $h_{1}, \cdots$, $h_{n+1}$ satisfy (i)-(viii) above. This completes the induction.

Now set $g=\sum_{n=1}^{\infty} g_{n}$. Then

$$
\begin{aligned}
F \circ\left(\mu+g_{n}\right) & =\left(\delta_{0}-h_{n}\right) * F \circ\left(\mu+g_{n}\right)+h_{n} * F \circ\left(\mu+g_{n}\right) \\
& =\left(\delta_{0}-h_{n}\right) * F \circ \mu+h_{n} * F \circ(\mu+g) .
\end{aligned}
$$

This now yields the absurdity of (7). 
Examples of algebras $X$ which satisfy (i)-(iii) are easily given: Let $E$ be a perfect compact independent subset of $G$. Such a set exists if $G$ is not discrete $[2,5.2 .2]$. Let $X$ be the closed subalgebra of $B(\Gamma)$ which is generated by $A(\Gamma), \hat{\delta}_{0}$, and the transforms of continuous measures supported on $E \cup-E$. One need only apply [2, 5.3.2] to show that $X$ satisfies (iii).

Varopoulos [3] has shown that if $G$ is not discrete, then only entire functions operate on $B_{0}(\Gamma)=\{\hat{\mu}: \hat{\mu} \in B(\Gamma), \hat{\mu}$ vanishes at infinity $\}$. $B_{0}(\Gamma) \cup\left\{\hat{\delta}_{0}\right\}$ is a closed subalgebra of $B(\Gamma)$ which satisfies (i)-(iii). Thus our Theorem 1 yields a variant of Varopoulos' result.

This comprises a portion of the author's doctoral dissertation at the Massachusetts Institute of Technology. The author is grateful to Professor Kenneth Hoffman for his advice and encouragement.

\section{BIBLIOGRAPHY}

1. I. M. Gelfand, Normierte ringe, Mat. Sb. 9(51) (1941), 3-24.

2. W. Rudin, Fourier analysis on groups, Wiley, New York, 1962.

3. N. Th. Varopoulos, The functions that operate on $B_{0}(\Gamma)$ of a discrete group $\Gamma$, Bull. Soc. Math. France 93 (1965), 301-321.

Massachusetts Institute of Technology and NORTHWESTERN UNIVERSITY 